\title{
A Framework for Reduction of Principal Component Analysis based Algorithms using Residue Number System
}

\author{
Tajudeen N. Madandola \\ Dept. of Computer Science \\ Kw. St. College of Education \\ Oro. Nigeria
}

\author{
Kazeem A. Gbolagade \\ Dept. of Computer Science \\ Kw. St. University \\ Malete
}

\author{
Yusuf-Asaju Ayisat W. \\ Dept. of Computer Science \\ University of Ilorin \\ Nigeria
}

\begin{abstract}
The level of criminality in Nigeria is at alarming rate which leads to daily development of various security skills used in identification and verification of offenders. Face Recognition (FR) which is one of the security skills is a biometric system used to identify or verify a person from a digital image. The dimensionality reduction is a most essential duty in the domain of FR. An algorithm that is used most of the times in FR for dimensionality reduction is Principal Component Analysis (PCA). PCA is a technique that can be used for dimensionality reduction but the major challenge of PCA is that it is a time-consuming data mining algorithm. Reducing the execution time of PCA has been a research significant topic in pattern recognition and computer vision. This paper presents a framework for execution time reduction of Principal Component Analysis based Algorithms focusing on CRT form of Residue Number System. Yale database and another new dataset is created containing 120 face images of 40 persons frontal faces with 3 images of each individual for the experiment.The proposed approach would decrease the execution time of PCA algorithm base algorithms.
\end{abstract}

\section{Keywords}

Residue Number System, Eigenfaces, Euclidean distance, principal component analysis, dimensionality reduction

\section{INTRODUCTION}

Safety defies as fronted to the utilization of numerous procedures of identification and verification since beginning of the world. Tribal marks, names, intonations and so on were used. Also, passwords (knowledge-based scheme) and ID cards (token-based schemes) have been used to validate the identity of an individual intending to access the services offered by an application. (e.g. online transaction). Face recognition technology has received laudable acceptance by both prosecution and non-prosecution agencies. Hefty datasets are progressively common and are often demanding to interpret. The method of decreasing the dimensionality of such datasets, increasing interpretability but at the same time minimizing information loss is called Principal Component Analysis (PCA) [6]. It is also known as Karhunen Loeve expansion (KLE). PCA is a classical feature extraction and data representation method broadly used in the areas of pattern recognition and computer vision. [10] first used PCA to efficiently represent pictures of human faces. The two of them claimed that any face image might be reconstructed almost as a weighted sum of a small collection of images that define a facial basis (eigenimages), and a mean image of the face. Within this context, [13] presented the well-known Eigenfaces method for face recognition in 1991. Since then
PCA has been widely investigated and has become one of the most successful approaches in face recognition [15],[19]. [9],[8] discussed the problem of the dimensionality of the "face space" when eigen-faces are used for representation. [18] tried to account for the arbitrary effects of illumination in PCA-based vision systems by generating an analytically closed form formula of the covariance matrix for the situation with a unusual lighting condition and later generalizing to an arbitrary illumination via an illumination equation.

However, [17] revealed that PCA could not apprehension even the simplest invariance unless this information is explicitly provided in the training data. They suggested a technique known as elastic bunch graph matching to overwhelm the weaknesses of PCA. Nowadays, two PCAassociated approaches, Independent Component Analysis (ICA) and Kernel Principal Component Analysis (Kernel PCA) have been of broad concern. [3] proposed using ICA for face representation and found that it was better than PCA when cosines were used as the similarity measure (however, their performance was not ominously different if the Euclidean distance is employed). PCA wishes to attain the most compact representations of the high-dimensional data under the sense of least square reconstruction error. [10] used PCA to represent human faces for the first time. Therefore, Turk and Pentland proposed a famous PCA-based face recognition method called eigenfaces [20]. Nevertheless, this does not imply that the eigenvectors can be evaluated exactly in this way since the eigenvectors are statistically determined by the covariance matrix, no matter what technique is adopted for obtaining them. Also, the main problem of PCA is that it is computer intensive algorithm; therefore improving the time of PCA is a research concern as shown in Figure 1. The experiment will be carried out using two different databases; a database was created containing 120 face images of 40 persons frontal faces with 3 images of each individual and Yale database which contains frontal gray scale face images of 15 people, with 11 face images of each subject. The researchers present a framework for improving the computational time and Memory usage of PCA algorithm. 


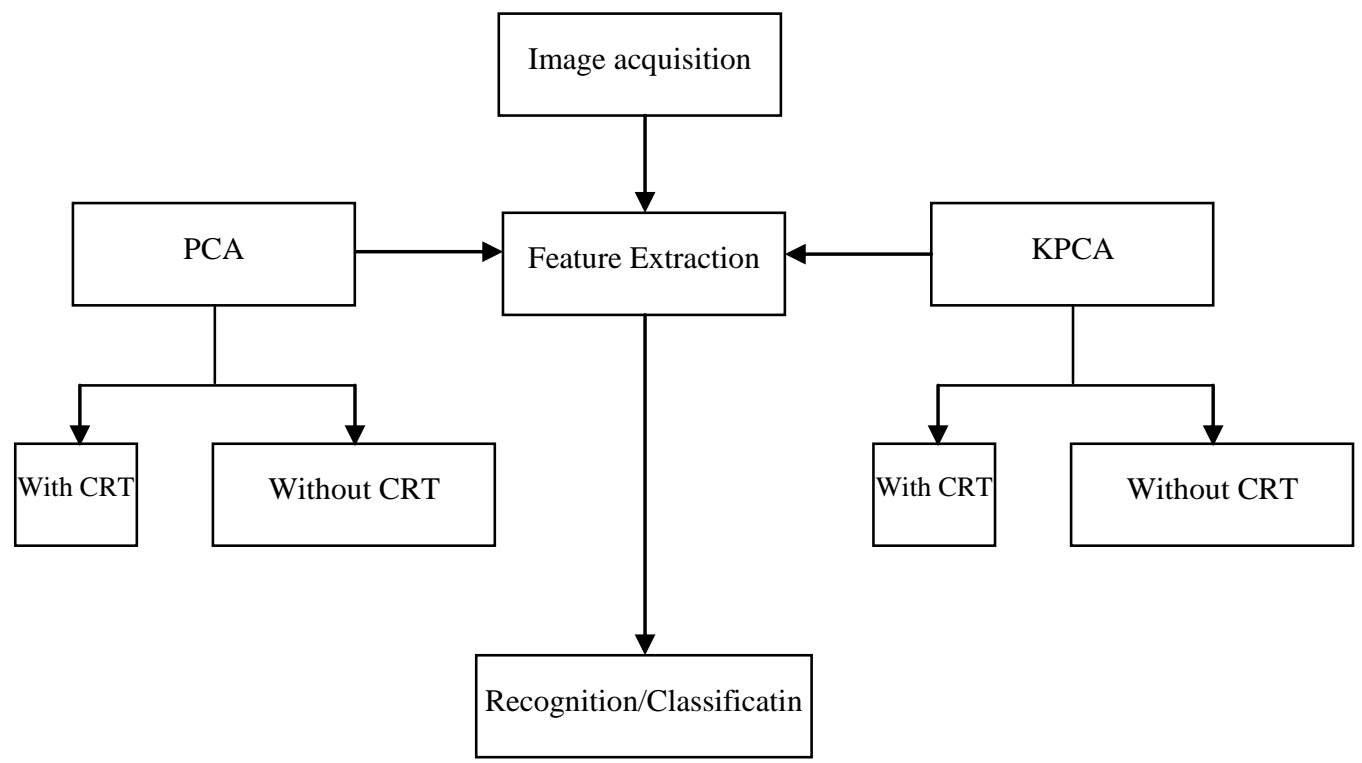

Figure 1: Research Proposed Framework

\section{LITERATURE REVIEW}

\subsection{Related Works}

The use of face recognition started in the 1960's with a semi automated system. The researchers made marks on photographs to locate the key features on used part such as eyes, ears, noses, and mouths. Later distances and ratios were computed from those marks to a common reference point and compared to reference data. In the early 1970's [5] created a system of 21 subjective markers such as hair colour and lip thickness. This demonstrated harder to automate due to the subjective nature of many of the measurements still made completely by hand. Another approach is the Connectionist approach [11], which seeks to classify the human face using a combination of both range of gestures and a set of identifying markers. The approach is usually implemented using 2dimensional pattern recognition and neural net principles. Often this approach involves a huge number of training faces to achieve decent accuracy; for that reason it has yet to be implemented on a large scale. The first fully automated system [22] to be developed utilized very general pattern recognition. It matched faces to a generic face model of anticipated features and created a series of patterns for an image relative to this model. This method is principally statistical and relies on histograms and the gray scale value.

Present days researchers used the eigenface approach for face recognition which was presented by [10] at Brown University. The method works by analyzing face images and computing eigenface [14] which are faces composed of eigenvectors. The assessment of eigenface is used to recognize the presence of a face and its identity. [15] developed a system that involved five step process. The system starts to initialize by feeding it a set of training images of faces. It is used to describe the face space which is set of images that are face like. Later when a face is encountered it calculates an eigenface for it. It would be determined whether the image presented is a face or not by comparing it with known faces and using some statistical analysis. If an image is determined to be a face the system will determine whether it recognizes the identity of it or not. The possible concluding step is that if an unknown face is seen repeatedly, the system can learn to recognize it, as shown in Figure 2.

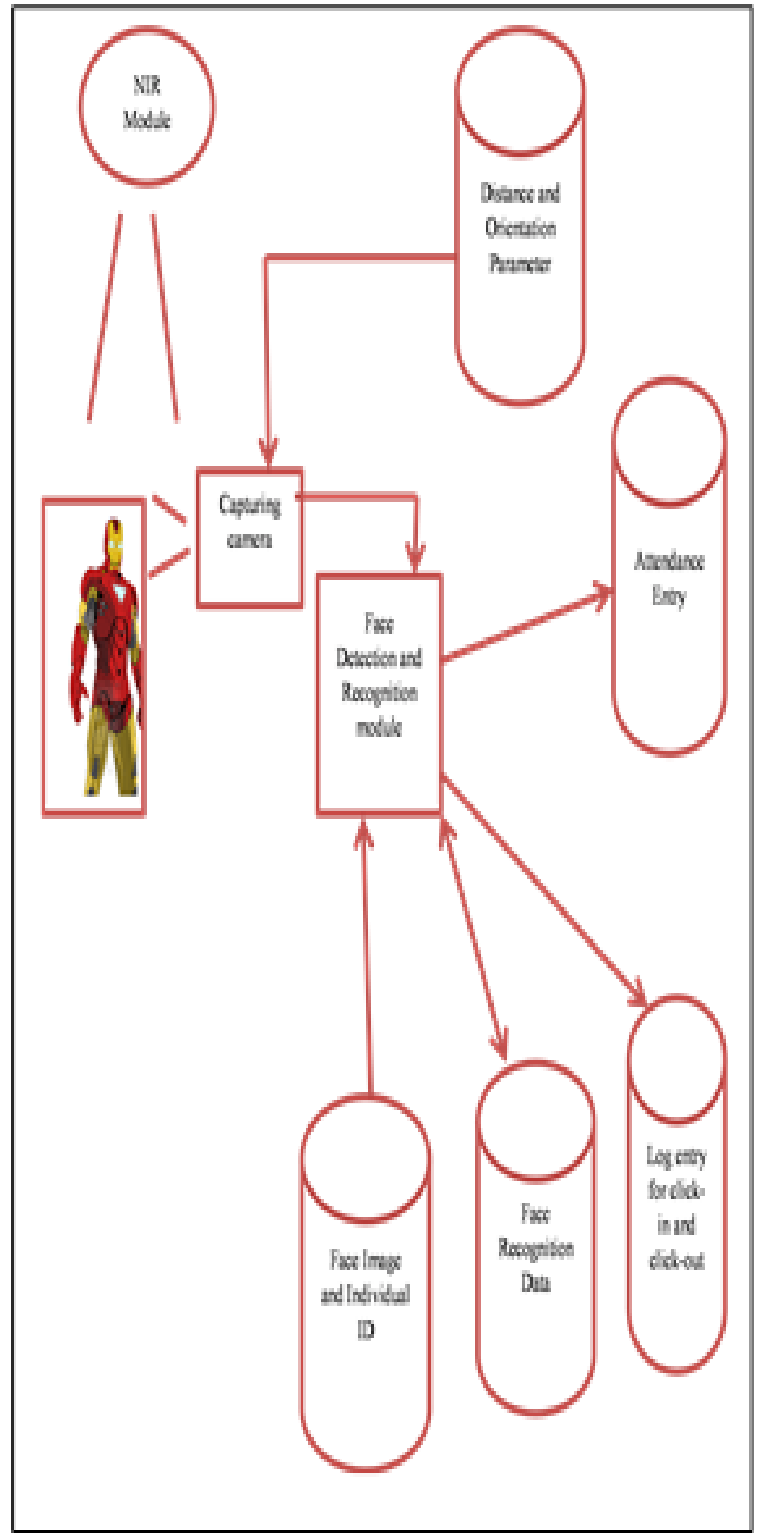

Figure 2: Overview of face recognition system [15]. 


\subsection{PCA (Principal Component Analysis)}

PCA technique has been broadly used in applications such as face recognition and image compression. It is a usual technique for locating patterns in data, and expressing the data as eigenvector to highlight the similarities and differences between different data. The below procedures review the PCA activity:

1. Represent training data set as $\{\mathrm{N} 1, \mathrm{~N} 2, \ldots \mathrm{NM}\}$. And average is Age:

$$
\begin{aligned}
& V_{x}=\phi \Lambda \phi^{T} \\
& V_{x}=\frac{1}{m} \sum_{i=1}^{m} x i x i^{T}
\end{aligned}
$$

$\phi=\left[\emptyset_{1}, \ldots . \varnothing_{\mathrm{n}}\right]$ is the eigenvector matrix of $V_{x} . \Lambda$ is a diagonal matrix, the eigenvalues $\lambda_{1}, \ldots, \lambda_{\mathrm{n}}$ of $V_{x}$ are located on its main diagonal. $\lambda_{\mathrm{i}}$ is the variance of the data projected on $\emptyset_{\mathrm{i}}$.

2. Every sample in the training data set varies from by the vector $\mathrm{Yi}=\mathrm{Ni}$-Age. The covariance matrix obtained as:

$K o v=\frac{1}{M} \sum_{i=1}^{M} Y i . Y i^{T}$

3. Pick M' significant eigenvectors of and compute the weight vectors $W i b$ the training data set, where $\mathrm{b}$ varies from 1 to $\mathrm{M}^{\prime}$.

$W_{i b}=E_{b}^{T} \cdot\left(N_{i}-A g e\right), \forall i, b$

\subsection{Residue Number System (RNS)}

It is a non-weighted number system that offers carry-free, parallel, high speed, protected and fault tolerant arithmetic operations [2]. The most primitive RNS hardware based was manufactured in 1967. In order to heighten RNS benefits, scholars look for way of improving it and associated problems and at last discover suitable applications for RNS's features. It aids carry-limited, high-speed arithmetic and error detection as well as error correction applications (Bankas \& Gbolagade, 2014). It is defined by a set of $N$ integer constants, $\left\{m_{1}, m_{2}, m_{3}, \ldots, m_{N}\right\}$, called the moduli. Let $M$ be the least common multiple of all the $m_{i}$. Any arbitrary integer $X$ smaller than $M$ can be represented in the defined residue numeral system as a set of $N$ smaller integers $\left\{x_{1}, x_{2}, x_{3}, \ldots, x_{N}\right\}$ with $x_{i}=X$ modulo $m$ representing the residue class of $X$ to that modulus. Chinese Remainder Theorem (CRT) and Mixed Radix Conversion (MRC) are the main two forms of RNS. $\mathrm{MRC}$ is an alternative scheme to CRT which does not involve the large modulo $\mathrm{M}$ calculations. MRC consents to a low complexity of $0(n)$ as compared to the CRT whose computation complexity of order $0(\mathrm{n} 3)$. The study will focus on CRT.

\subsection{Chinese Remainder Theorem (CRT)}

According to [12] the traditional CRT is defined as follows: for a moduli set $\left\{\mathrm{m}_{1}, \mathrm{~m}_{2}, \mathrm{~m}_{3}, \ldots, \mathrm{m}_{\mathrm{k}}\right\}$ with the dynamic range $\mathrm{M}=\prod_{i=1}^{k} m i$,

the residue number $\left(\mathrm{x}_{1}, \mathrm{x}_{2}, \mathrm{x}_{3}, \ldots, \mathrm{x}_{\mathrm{k}}\right)$ can be transformed into the decimal number $\mathrm{X}$,

as follows:

$$
\mathrm{X}=\left.\left.\left|\sum_{i=1}^{k} M_{i}\right| M_{i}^{-1} \mathcal{X}_{i}\right|_{m_{i}}\right|_{M}
$$

Where $\mathrm{M}=\prod_{i=1}^{k} m i, \mathrm{M}_{\mathrm{i}}=\frac{M}{m i}$, and $M_{i}^{-1}$ is the multiplicative inverse of $\mathrm{M}_{\mathrm{i}}$ with respect to $\mathrm{m}_{\mathrm{i}}$. CRT is mainly based on the algorithm of linear congruencies. The system of linear congruencies $\mathrm{a} \equiv \mathrm{b}(\bmod \mathrm{m})$ can be reduced to a set of a $\equiv \mathrm{b}\left(\bmod \mathrm{m}_{\mathrm{i}}\right)$, with : $1 \leq \mathrm{i} \leq \mathrm{k}$

$\mathrm{x} \equiv\left[\mathrm{a}_{1} \mathrm{M}_{1} \mathrm{y}_{1}+\mathrm{a}_{2} \mathrm{M}_{2} \mathrm{y}_{2}+\ldots+\mathrm{a}_{\mathrm{k}} \mathrm{M}_{\mathrm{k}} \mathrm{y}_{\mathrm{k}}\right](\bmod \mathrm{M})$ with $\mathrm{M}=\mathrm{m}_{1}$

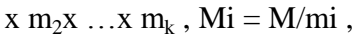

yi $\equiv\left(M_{i}\right)^{-1}\left(\bmod m_{i}\right)$ and $y_{i}$ integer which verifies: $y_{i} M_{i}$ $\equiv 1\left(\bmod \mathrm{m}_{\mathrm{i}}\right)$.

(Two numbers are considered to be relatively prime if their greatest common divisor is 1 ). If the equation set does not meet the restrictions (no co-primes) to be solved by the traditional CRT method, then the algorithm should convert it to a new set of equations, where the moduli are relatively prime.

\section{METHODOLOGY}

\subsection{Research Proposed Framework}

The Research proposed framework for the research as shown in Figure 1, will be used as the organization to be followed in solving the research problems. It will start from image acquisition from two face databases (Yale Database and a proposed setup database). Then Principal Component Analysis (PCA) and Kernel Principal Component Analysis (KPCA) will be employed as face feature extraction algorithms. Each of the feature extraction algorithms will be used separately and face recognition accuracy and computational time results will be acquired using Training time, Testing time as performance metrics. Subsequent Chinese Remainder Theorem will be fused to each of the algorithms separately then determine the recognition accuracy and Computational time. The first results (PCA only) shall be compared with PCA with CRT (PCA-CRT) and the KPCA results will be also compared with KPCA-CRT. The experiment will be performed on each of the database.

\subsection{Design Databases}

Two different databases will be used for the experiment. The first database will be local database (facial database created by the researchers) and Standard database (Yale database). For the first database, digital camera was used to capture 40 persons frontal faces with 3 images of each individual under different lighting, facial expressions, occulations, environment and time while the second database contains frontal gray scale face images of 15 people, with 11 face images of each subject giving a total of 165 images, as shown in Figure 3. 

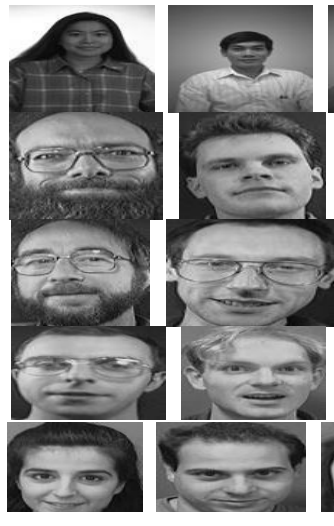

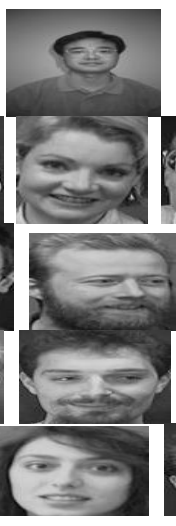

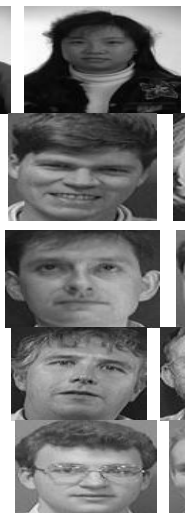

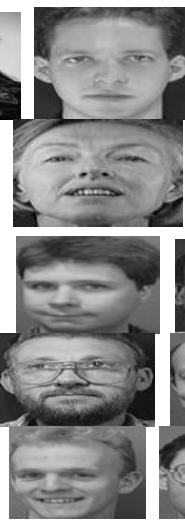

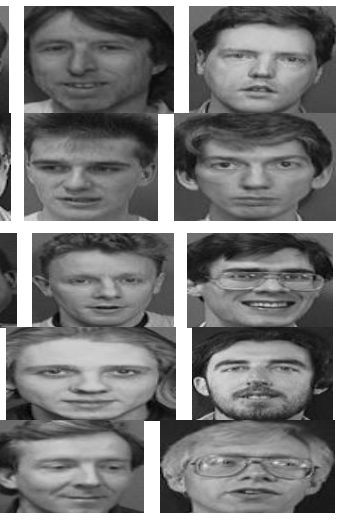

Figure 3: Part of images in Yale database [21].

\section{CONCLUSION}

Improving the computational Time and Memory usage of PCA algorithm is the main focus of this paper. PCA is a popular and widely used FR algorithm for dimensionality reduction and it is one of the most successful face feature extraction. This algorithm is known to be slow as compared to other FR algorithm. Moreso, having realized that delay in authenticating a criminal/suspect degraded the system performance and acceptability. Therefore, Chinese remainder theorem (CRT) form of Residue Number System is proposed in this paper to improve the performance speed of PCA. Future work will be to implement this proposed scheme, evaluate the accuracy performance of the system and compared with the traditional PCA algorithm.

\section{ACKNOWLEDGMENTS}

Researchers sincere appreciations to International Journal Computer and Application for publishing this paper timely.

\section{REFERENCES}

[1] Bankas, E. K., and Gbolagade, K. A. 2014. A new efficient RNS reverse converter for the 4-Moduli Set $\left\{2^{\text {n }}\right.$, $\left.2^{\mathrm{n}}+1,2^{\mathrm{n}}-1,2^{2 \mathrm{n}+1}-1\right\}$. International Journal of Computer, Electrical, Automation, Control and Information Engineering. 8(2): 328 - 332.

[2] Barati, A., Movaghar, A., and Sabaei, M. 2014. Energy efficient and high-speed error control scheme for real time wireless sensor networks. International Journal of Distributed Sensor Networks. 1-9.

[3] Draper, K.B., Bartlett, M.S. and Beveridge, J. R. 2002. "Recognizing Faces with PCA and ICA," Computer Vision and Image Understanding: special issue on face recognition, in press.

[4] Gbolagade, K. A. 2010. Effective Reverse Conversion in Residue Number System Processors. PhD Thesis. The Netherlands.

[5] Goldstein, A.J., Harmon, L. D., and Lesk, A. B. 2002. "Identification of Human Faces," in Proceeding of IEEE Conference on Computer Vision and Pattern Recognition. 59: $748-760$.

[6] Jolliffe I.T and Cadima J. 2016. Principal component analysis: A review and recent developments. Phil. Trans. R. Soc. A 374: 20150202.

[7] Kirby, M. and Sirovich, L. 1990. "Application of the KL Procedure for the Characterization of Human Faces," IEEE Trans. Pattern Analysis and Machine Intelligence. 12(1): 103-108.
[8] Li, Y., Fermuller, C., Aloimonos, Y. and Hui, J. 2010. "Learning shift-invariant sparse representation of actions," in Proc. IEEE Conf. CVPR: 2630-2637.

[9] Penev, P.S. and Sirovich, L. 2000, "The Global Dimensionality of Face Space," Proc. Fourth IEEE Int'l Conf. Automatic Face and Gesture Recognition. 264270.

[10] Sirovich, L. and Kirby, M. 1988. "Low-Dimensional Procedure for Characterization of Human Faces," J. Optical Soc. Am. 4: 519-524.

[11] Stan Z. Li and Anil K. Jain. 1999. "Handbook of Face Recognition" Springer publications.

[12] Szabo, N. and Tanaka, R. 1967. Residue Arithmetic and its Application to Computer Technology. MC-Graw-Hill, New York.

[13] Turk, M. A. and Pentland, A. 1991a. "Face Recognition Using Eigenfaces," in Proc. IEEE Conference on Computer Vision and Pattern Recognition. 586-591.

[14] Turk, M.A. and Pentland, A. 1991b. " Eigenfaces for Recognition." vision and Modeling

Group, The Media Laboratory, Massachusetts Institute of Technology.

[15] Turk, M.A. and Pentland, A. 1991c. "Eigenfaces for Recognition,” J. Cognitive Neuroscience. 3(1): 71-86.

[16] Valentin, D., Abdi, H., O’Toole, A.J. and Cottrell, G.W. 1994. "Connectionist Models of Face Processing: a Survey," Pattern Recognition. 27(9): 1209-1230.

[17] Wiskott, L., Fellous, J.M., Kru"ger, N. and Malsburg, C. von der. 1997. "Face Recognition by Elastic Bunch Graph Matching," IEEE Trans. Pattern Analysis and Machine Intelligence. 19(7): 775-779.

[18] Zhao, L. and Yang, Y. 1999. "Theoretical Analysis of Illumination in PCA-Based Vision Systems," Pattern Recognition. 32(4): 547-564.

[19] Zhao, Q., Zhou, G., Adali, T. Zhang, L. and Cichocki, A. 2013. "Kernelization of tensor-based models for multiway data analysis," IEEE Signal Process. Mag.. 30(4): 137-148.

[20] Zhihui, Lai, Yong Xu, Qingcai Chen, Jian Yang. and David Zhang. 2014. Multilinear Sparse Principal Component Analysis. IEEE Transactions on Neural Networks and Learning System.

[21] The Yale database, Available: http://cvc.yale.edu/

[22] The pattern recognition Generic images, Available http.www.face-rec.org 